Bull. Austral. Math. Soc.

$47 \mathrm{H} 10,54 \mathrm{H} 25$

VOL. 59 (1999) [481-493]

\title{
A KKM TYPE THEOREM AND ITS APPLICATIONS
}

\author{
LAI-JIU LIN
}

In this paper we establish a generalised KKM theorem from which many well-known $K K M$ theorems and a fixed point theorem of Tarafdar are extended.

\section{INTRODUCTION}

In [6], Knaster, Kuratoaski and Mazurkiewicz established the well known $K K M$ theorem on the closed cover of a simplex. In [4], Ky Fan generalised the $K K M$ theorem to a subset of any topological vector space. There are many generalisations and many applications of this theorem.

In this paper, we establish a generalised $K K M$ theorem on a generalised convex space as follows:

THEOREM 1. Let $(X, D ; \Gamma)$ be a $G$-convex space, $Y$ a Hausdorff space and $T \in$ $G-K K M(X, Y)$ be compact, and $G: D \rightarrow 2^{Y}$. Suppose that

(1.1) for each $x \in D, G x$ is compactly closed in $Y$; and

(1.2) for any $N \in\langle D\rangle, T\left(\Gamma_{N}\right) \subseteq G(N)$.

Then $\overline{T(X)} \cap \cap\{G x: x \in D\} \neq \emptyset$.

Applying Theorem 1, we extend many well-known generalised $K K M$ theorem, and we give a unified treatment of these theorems (see $[5,7,9,10,12,14,15,16])$. We also obtain some equivalent forms of Theorem 1 and extend a fixed point theorem of Tarafdar [15].

\section{PRELIMINARIES}

Let $X, Y$ and $Z$ be nonempty sets; $2^{Y}$ will denote the power set of $Y$. Let $F: X \rightarrow 2^{Y}$ be a set-valued map, $A \subseteq X, B \subseteq Y$ and $y \in Y$. We define

$$
\begin{aligned}
F^{-}(B) & =\{x \in X: F(x) \cap B \neq \emptyset\}, \quad F^{-}(y)=\{x \in X: y \in F(x)\}, \\
F(A) & =\bigcup\{F(x): x \in A\}, \quad G_{r}(F)=\{(x, y): y \in F(x), x \in X\} .
\end{aligned}
$$

Received 8th December, 1998

This research was supported by the National Science Council of the Republic of China.

Copyright Clearance Centre, Inc. Serial-fee code: 0004-9729/99 \$A2.00+0.00. 
For topological spaces $X$ and $Y$, a map $F: X \rightarrow 2^{Y}$ is said to be upper semicontinuous if the set $F^{-}(A)$ is closed in $X$ for each closed subset $A$ of $Y$. $F$ is said to be closed if $G_{r}(F)$ is a closed subset of $X \times Y$, and $F$ is said to be compact if $\overline{F(X)}$ is a compact subset of $Y$. A subset $B$ of $Y$ is said to be compactly closed (compactly open) if for each compact subset $K$ of $Y$, the set $B \cap K$ is closed (open) in $K$.

Given two set-valued maps $F: X \rightarrow 2^{Y}, G: Y \rightarrow 2^{Z}$ the composite $G F: X \rightarrow 2^{Z}$ is defined by $G F(x)=G(F(x))$ for $x \in X$. Let $\mathbb{X}$ be a class of set-valued maps. We write $\mathbb{X}(X, Y)=\left\{T: X \rightarrow 2^{Y} \mid T \in \mathbb{X}\right\}, \mathbb{X}_{c}(X, Y)=\left\{T_{n} T_{n-1} \cdots T_{1}: T_{i} \in \mathbb{X}, i=1,2, \ldots, n\right.$ for some $n\}$, that is, the set of finite composites of maps in $X$.

The following notion of an abstract class of set-valued maps was introduced by Park [10]. A class $U$ of set-valued maps is one satisfying the following:

(i) $U$ contains the class $\mathbb{C}$ of single-valued continuous functions;

(ii) each $T \in U_{c}$ is upper semicontinuous with compact values; and

(iii) for each polytope $P$, each $T \in U_{c}(P, P)$ has a fixed point.

We write $U_{c}^{\kappa}(X, Y)=\left\{T: X \rightarrow 2^{Y} \mid\right.$ for any compact subset $K$ of $X$, there is $F \in U_{c}(K, Y)$ such that $F(x) \subseteq T(x)$ for each $\left.x \in K\right\}$. Each $F \in U_{c}^{\kappa}$ is said to be admissible.

Let $X$ be a convex set in a vector space and $D$ a nonempty subset of $X$. Then $(X, D)$ is called a convex space if the convex hull of any nonempty finite subset of $D$ is contained in $X$ and $X$ has the topology that induces the Euclidean topology on the convex hull of its finite subsets. For a nonempty subset $D$ of $X$, let $\langle D\rangle$ denote the set of all nonempty finite subsets of $D$. Let $\triangle_{n}$ denote the standard $n$-simplex with vertices $e_{1}, e_{2}, \ldots, e_{n+1}$, where $e_{i}$ is the $i$ th unit vector in $\mathcal{R}^{n+1}$, that is $\Delta_{n}=\left\{u \in \mathcal{R}^{n+1}: u=\sum_{i=1}^{n+1} \lambda_{i}(u) e_{i}, \lambda_{i}(u) \geqslant 0\right.$, $\left.\sum_{i=1}^{n+1} \lambda_{i}(u)=1\right\}$

A generalised convex space [12] or a $G$-convex space $(X, D ; \Gamma)$ consists of a topological space $X$, a nonempty subset $D$ of $X$ and a function $\Gamma:\langle D\rangle \rightarrow 2^{X}$ with nonempty values such that

1. for each $A, B \in\langle D\rangle, A \subset B$ implies $\Gamma(A) \subseteq \Gamma(B)$ and

2. for each $A \in\langle D\rangle$, with $|A|=n+1$, there exists a continuous function $\phi_{A}: \triangle_{n} \rightarrow \Gamma(A)$ such that $J \in\langle A\rangle$ implies $\phi_{A}\left(\triangle_{J}\right) \subset \Gamma(J)$, where $\triangle_{J}$ denotes the face of $\triangle_{n}$ corresponding to $J \in\langle A\rangle$.

We see from [12] that a convex subset of a topological vector space, Lassonde's convex space, $S$-contractible space, $H$-space, a metric space with Michael's convex structure, Komiya's convex space, Bielawski's simplicial convexity, Joo's pseudoconex space are examples of $G$-convex spaces.

For a $G$-convex space $(X, D ; \Gamma)$, a subset $C$ of $X$ is said to be $G$-convex if for each $A \in\langle D\rangle, A \subseteq C$ implies $\Gamma(A) \subset C$. We sometimes write $\Gamma(A)=\Gamma_{A}$ for each $A \in\langle D\rangle$. 
Defintion 1: Let $(X, D ; \Gamma)$ be a $G$-convex space, $T: X \rightarrow 2^{Y}$ and $S: D \rightarrow 2^{Y}$ be two set-valued maps such that $T\left(\Gamma_{A}\right) \subset S(A)$ for each $A \in\langle D\rangle$. Then we call $S$ a generalised $G-K K M$ map with respect to $T$. Let $T: X \rightarrow 2^{Y}$ be a set-valued map. $T$ is said to have the $G-K K M$ property if whenever $S: D \rightarrow 2^{Y}$ is any generalised $G-K K M$ map with respect to $T$, then the family $\{\overline{S x}: x \in D\}$ has the finite intersection property. We let $G-K K M(X, Y)=\left\{T: X \rightarrow 2^{Y} \mid T\right.$ has the $G-K K M$ property $\}$. If $(X, D)$ is a convex space, and $\Gamma_{A}=$ Co $A$ is the convex hull of $A$, then $G-K K M(X, Y)=K K M(X, Y)$ as defined in [3].

LEMma 1. Let $(X, D ; \Gamma)$ be a $G$-convex space, and $Y$ a Hausdorff space. Then $U_{c}^{\kappa}(X, Y) \subseteq G-K K M(X, Y)$

Proof: Lemma 1 follows immediately from the corollary of [13, Theorem 2] and Definition 1.

Lemma 2. [1] Let $Y$ be a compact space and $F: X \rightarrow 2^{Y}$ be closed. Then $F$ is upper semicontinuous.

Lemma 3. [1] Let $F: X \rightarrow 2^{Y}$ be upper semicontinuous with compact values from a compact space $X$ to $Y$. Then $F(X)$ is compact.

Lemma 4. [1] Let $X \rightarrow 2^{Y}$ be upper semicontinuous with closed values. Then $F$ is closed.

LEMma 5. [3] Let $X$ be a convex subset of a linear space, and $Y$ be a topological space. Then $T \in K K M(X, Y)$ if and only if $\left.T\right|_{P} \in K K M(P, Y)$ for each polytope $P$ in $X$.

Lemma 6. Let $X$ be a convex subset of a linear space, $Y$ a topological space, $A$ a convex subset of $X$, and $T \in K K M(X, Y)$. Then $\left.T\right|_{A} \in K K M(A, Y)$.

Proof: Let $P$ be any polytope in $A$. Since $T \in K K M(X, Y)$, it follows from Lemma 5 that $\left.T\right|_{P} \in K K M(P, Y)$. But $\left.\left(\left.T\right|_{A}\right)\right|_{P}=\left.T\right|_{P} \in K K M(P, Y)$. Again by applying Lemma 5, $\left.T\right|_{A} \in K K M(A, Y)$.

A nonempty topological space is acyclic if all its reduced Cẽch homology groups over rationals vanish. In particular, any contractible space is acyclic, any convex or starshaped space is acyclic. For a convex space $Y, k(Y)$ denotes the set of all nonempty compact convex subsets of $Y, k a(Y)$ denotes the set of all compact acyclic subsets of $Y$ and $V(X, Y)=\{T \mid T: X \rightarrow k a(Y)$ is upper semicontinuous $\}$. Throughout this paper, all topological spaces are assumed to be Hausdorff.

\section{Main Results}

We prove a generalised $G-K K M$ theorem which gives a unified approach to $K K M$ type theorems. 
TheOREM 1. Let $(X, D ; \Gamma)$ be a $G$-convex space, $Y$ a Hausdorff space and $T \in$ $G-K K M(X, Y)$ be compact, $G: D \rightarrow 2^{Y}$. Suppose that

(1.1) for each $x \in D, G x$ is compactly closed in $Y$; and

(1.2) for any $N \in\langle D\rangle, T\left(\Gamma_{N}\right) \subseteq G(N)$.

Then $\overline{T(X)} \cap \cap\{G x: x \in D\} \neq \emptyset$.

Proof: Since $T$ is compact, there exists a compact set $K$ of $Y$ such that $T(X) \subseteq K$. From this, we see that $\overline{T(X)}$ is compact. For each $x \in D$, let $S x=\overline{T(X)} \cap G x$, then it follows from (1.1) that $S x$ is closed in $\overline{T(X)}$ for each $x \in D$. By (1.2), we see that for any $N \in\langle D\rangle, T\left(\Gamma_{N}\right)=T\left(\Gamma_{N}\right) \cap \overline{T(X)} \subseteq G(N) \cap \overline{T(X)}=S(N)$. Hence $S$ is $G-K K M$ with respect to $T$. It follows that $\{S x: x \in D\}=\{\overline{S x}: x \in D\}$ has the finite intersection property. Since $\overline{T(X)}$ is compact and $\{S x: x \in D\}$ is a family of closed subsets in $\overline{T(X)}$, we have $\bigcap\{S x: x \in D\} \neq \emptyset$. Therefore $\overline{T(X)} \cap \cap\{G x: x \in D\} \neq \emptyset$.

REMARK 1. In Theorem 1 , if the condition $T \in G-K K M(X, Y)$ is compact is replaced by the condition that $T \in U_{c}^{\kappa}(X, Y)$ and $X$ is compact, then we obtain the following corollary.

Corollary 1. Let $(X, D ; \Gamma)$ be a compact $G$-convex space, $Y$ a Hausdorff space, and $T \in U_{c}^{\kappa}(X, Y)$. Suppose that

(C1.1) for each $x \in D, G x$ is compactly closed in $Y$; and

(C1.2) for each $N \in\langle D\rangle, T\left(\Gamma_{N}\right) \subseteq G(N)$.

Then $\overline{T(X)} \cap \cap\{G x: x \in D\} \neq \emptyset$.

Proof: Since $X$ is compact and $T \in U_{c}^{\kappa}(X, Y)$, there exists $T^{\prime} \in U_{c}(X, Y)$ such that $T^{\prime} x \subseteq T x$ for all $x \in X$. Since $T^{\prime}$ is upper semicontinuous with compact-values on $X$, it follows from Lemma 3 that $T^{\prime}(X)$ is compact. Hence $T^{\prime} \in U_{c}(X, Y) \subset K K M(X, Y)$ is compact. By (C1.2), for each $N \in\langle D\rangle, T^{\prime}\left(\Gamma_{N}\right) \subseteq G(N)$. Then all the conditions for Theorem 1 are satisfied and it follows from Theorem 1 that $\overline{T^{\prime}(X)} \cap \cap\{G x: x \in D\} \neq \emptyset$. Therefore $\overline{T(X)} \cap \cap\{G x: x \in D\} \neq \emptyset$.

Theorem 2. Let $(X, D)$ be a convex space, $Y$ a Hausdorff space and $G: D \rightarrow 2^{Y}$, $T \in U_{c}^{\kappa}(X, Y)$ be set-valued maps satisfying the following

(2.1) for each $N \in\langle D\rangle, T(\operatorname{Co} N) \subseteq G(N)$; and

(2.2) for each $N \in\langle D\rangle$, and each $x \in N, G x \cap T(\operatorname{Co} N)$ is relatively closed in $T(\operatorname{Co} N)$.

Then, for each $N \in\langle D\rangle, T(\operatorname{Co} N) \cap \cap\{G x: x \in N\} \neq \emptyset$.

Proof: Let $\widetilde{N} \in\langle D\rangle$, and $Z=$ Co $\widetilde{N}$. Since $T \in U_{c}^{\kappa}(X, Y)$ and $Z$ is compact, there exists $F \in U_{c}(Z, Y)$ such that $F x \subseteq T x$ for each $x \in Z$. As $F$ is upper semicontinuous with compact values, it follows from Lemma 4 that $F(Z)$ is compact and $F$ is compact. Let $G_{1}: \widetilde{N} \rightarrow 2^{Y}$ be given by $G_{1} x=G x \cap F(Z)$ for $x \in \widetilde{N}$. Then for each $N \in\langle\widetilde{N}\rangle$, 
$F(\operatorname{Co} N)=F(\operatorname{Co} N) \cap F(Z) \subseteq T(\operatorname{Co} N) \cap F(Z) \subseteq G(N) \cap F(Z)=G_{1}(N)$. By (2.2), for each $x \in \widetilde{N}, G x \cap T(Z)=A x \cap T(Z)$, where $A: \widetilde{N} \rightarrow 2^{Y}, A x$ is closed for each $x \in \widetilde{N}$. Hence for each $x \in \widetilde{N}, G_{1} x=G x \cap F(Z)=G(x) \cap T(Z) \cap F(Z)=A x \cap T(Z) \cap$ $F(Z)=A x \cap F(Z)$ is closed in $Y$. This shows that for each $x \in \widetilde{N}, G_{1} x$ is compactly closed in $Y$. We see $F \in U_{c}(Z, F(Z)) \subseteq K K M(Z, F(Z))$. Replacing $(D, X, Y, T, G)$ by $\left(\widetilde{N}, Z, F(Z), F, G_{1}\right)$ in Theorem 1 , shows that $\overline{F(Z)} \cap \cap\left\{G_{1} x: x \in \widetilde{N}\right\} \neq \emptyset$. This implies $\overline{T(Z)} \cap \cap\{G x: x \in \widetilde{N}\} \neq \emptyset$. Since $\widetilde{N} \in\langle D\rangle$ is arbitary, this completes the proof.

Corollary 2. Let $X$ be a nonempty subset of a vector space, and $G: X \rightarrow 2^{Y}$, $T: \operatorname{Co} X \rightarrow k a(Y)$ set-valued maps satisfying the following

(C2.1) for each $N \in\langle X\rangle, T(\mathrm{Co} N) \subseteq G(N)$;

(C2.2) for each $N \in X,\left.T\right|_{\text {CoN }}$ is upper semicontinuous, where Co $N$ is endowed with the Euclidean simplex topology; and

(C2.3) for each $N \in\langle X\rangle$, and each $x \in N, G x \cap T(\operatorname{Co} N)$ is relatively closed in $T(\operatorname{Co} N)$.

Then for each $N \in\langle X\rangle, T($ Co $N) \cap \cap\{G x: x \in N\} \neq \emptyset$.

Proof: Let $\widetilde{X} \in\langle X\rangle$. By (C2.2), (Co $\widetilde{N}, \widetilde{N})$ is a convex space and $\left.T\right|_{\text {Co } \tilde{N}} \epsilon$ $V($ Co $\widetilde{N}, Y) \subseteq U_{c}^{\kappa}($ Co $\widetilde{N}, Y)$. Then all conditions of Theorem 2 are satisfied and Corollary 2 follows immediately from Theorem 2.

Applying Theorem 1, we generalise Fan [5, Theorem 6] and we improve [3, Theorem 8].

THEOREM 3. Let $X$ be a convex space, $Y$ a Hausdorff space and $S: X \rightarrow 2^{Y}$, $T \in K K M(X, Y)$ maps satisfying the following conditions:

(3.1) for each compact subset $C$ of $X, \overline{T(C)}$ is a compact subset of $Y$;

(3.2) for each $x \in X, S x$ is compactly closed in $Y$;

(3.3) for each $N \in\langle X\rangle, T(\operatorname{Co} N) \subseteq S(N)$; and

(3.4) there exists a compact convex subset $X_{0}$ of $X$ and

$$
\bigcap\left\{S x: x \in X_{0}\right\} \subseteq K
$$

Then $\overline{T(X)} \cap \cap\{S x: x \in X\} \neq \emptyset$.

Proof: Suppose that $\overline{T(X)} \cap \cap\{S x: x \in X\}=\emptyset$. Since $K$ is compact, there exists a finite subset $\left\{x_{1}, x_{2}, \ldots, x_{n}\right\}$ of $X$ such that $K \subseteq(\overline{T(X)})^{c} \cup\left(\bigcup_{i=1}^{n} S^{c} x_{i}\right)$, where $S^{c} x=Y \backslash S x$. By (3.4), $K^{c} \subseteq \bigcup_{x \in X_{0}} S^{c} x_{i} \subseteq\left(\bigcup_{x \in X_{0}} S^{c} x\right) \cup(\overline{T(X)})^{c}$. If we let $X_{1}=\operatorname{Co}\left(X_{0} \cup\right.$ $\left.\left\{x_{1}, x_{2}, \ldots, x_{n}\right\}\right)$, then $X_{1}$ is a compact convex subset of $X$ and $Y=\left(\bigcup_{x \in X_{1}} S^{c} x\right) \cup(\overline{T(X)})^{c}$, that is, $\overline{T(X)} \cap \bigcap_{x \in X_{1}} S x=\emptyset$. We define $F: X_{1} \rightarrow 2^{Y}$ by $F x=S x \cap \overline{T\left(X_{1}\right)}, x \in X_{1}$. 
Then (a) for each $x \in X_{1}, F x$ is a closed subset of $\overline{T\left(X_{1}\right)}$, (b) for each $N \in\left\langle X_{1}\right\rangle$, $T($ Co $N) \subseteq F(N)$. Since $T \in K K M(X, Y)$, it follows from Lemma 6 and (3.1) that $\left.T\right|_{X_{1}} \in K K M\left(X_{1}, Y\right)$ is compact. By Theorem 1, we have $\overline{\left.T\right|_{X_{1}}\left(X_{1}\right)} \cap \cap\left\{S x: x \in X_{1}\right\} \neq \emptyset$. But $\left.T\right|_{X_{1}}\left(X_{1}\right) \subseteq T(X)$, so we have $\overline{T(X)} \cap \cap\left\{S x: x \in X_{1}\right\} \neq \emptyset$. This contradicts that $\overline{T(X)} \cap \cap\left\{S x: x \in X_{1}\right\}=\emptyset$. Therefore $\overline{T(X)} \cap \cap\{S x: x \in X\} \neq \emptyset$.

Remark 2. Theorem 3 improves [3, Theorem 8]. We prove Theorem 3 by applying Theorem 1, while [3, Theorem 8$]$ is proved by applying the $K K M$ property. From [3, Theorem 8] we only obtain the conclusion $\bigcap_{x \in X} S x \neq \emptyset$.

Corollary 3. [5] In a topological vector space, let $Y$ be a convex set and $\emptyset \neq$ $X \subset Y$. For each $x \in X$, let $F(x)$ be a relatively closed subset of $Y$ such that the convex hull of every finite subset $\left\{x_{1}, x_{2}, \ldots, x_{n}\right\}$ of $X$ is contained in the corresponding union $\bigcup_{i=1}^{n} F\left(x_{i}\right)$. If there is a nonempty subset $X_{0}$ of $X$ such that the interection $\bigcap_{x \in X_{0}} F(x)$ is compact, and $X_{0}$ is contained in a compact convex subset of $Y$, then $\bigcap_{x \in X} F(x) \neq \emptyset$.

Proof: Take $T(x)=\{x\}$ and $K=\bigcap_{x \in X_{0}} F(x)$; then Corollary 3 follows immediately.

Corollary 4. Let $X$ be a convex space, $Y$ a Hausdorf space, and $S: X \rightarrow 2^{Y}$, $T \in K K M(X, Y)$ maps satisfying the following

(C4.1) for each compact subset $C$ of $X, \overline{T(C)}$ is compact;

(C4.2) for each $x \in X, S x$ is compactly closed in $Y$;

(C4.3) for each $N \in\langle X\rangle, T(\mathrm{Co} N) \subseteq S(N)$; and

(C4.4) there is a nonempty subset $X_{0}$ of $X$ such that $X_{0}$ is contained in a compact convex subset $X_{1}$ of $X$ and $\bigcap_{x \in X_{0}} S x$ is a nonempty compact subset of $Y$.

Then $\overline{T(X)} \cap \cap\{S x: x \in X\} \neq \emptyset$.

Proof: If we take $K=\bigcap_{x \in X_{0}} S x$ in Theorem 3, then Corollary 4 follows immediately.

Theorem 4. Let $X$ be a convex space, $Y$ a Hausdorff space, $S: X \rightarrow 2^{Y}$, $T \in U_{c}^{\kappa}(X, Y)$ satisfying

(4.1) for each $x \in X, S x$ is compactly closed in $Y$;

(4.2) for each $N \in\langle X\rangle, T($ Co $N) \subseteq S(N)$; and

(4.3) there exists a nonempty subset $K$ of $Y$ and a nonempty subset $X_{0}$ of $X$ such that $X_{0}$ is contained in a compact convex subset $X_{1}$ of $X$ and $\cap\left\{S x: x \in X_{0}\right\} \subseteq K$.

Then $K \cap \overline{T(X)} \cap \cap\{S x: x \in X\} \neq \emptyset$. 
Proof: Let $N=\left\{x_{1}, x_{2}, \ldots, x_{n}\right\}$ be any finite subset of $X$, then it follows from (4.3) that $X_{2}=\mathrm{Co}\left(X_{1} \cup N\right)$ is a compact convex subset of $X$. By the assumption $T \in U_{c}^{\kappa}(X, Y)$, there exists $T^{\prime} \in U_{c}\left(X_{2}, Y\right)$ such that $T^{\prime} x \subseteq T x$ for all $x \in X_{2}$ and $T^{\prime}\left(X_{2}\right)$ is a compact subset of $Y$. Thus $T^{\prime} \in U_{c}\left(X_{2}, Y\right) \subseteq K K M\left(X_{2}, Y\right)$ is compact. Then all the conditions of Theorem 1 are satisfied. It follows from Theorem 1 that $\overline{T^{\prime}\left(X_{2}\right)} \cap \cap\left\{S x: x \in X_{2}\right\} \neq \emptyset$. Hence $\overline{T^{\prime}\left(X_{2}\right)} \cap \cap\left\{S x \cap \cap\left\{S x: x \in X_{1}\right\}: x \in N\right\} \neq \emptyset$. But $X_{0} \subset X_{1}$, hence $\bigcap\left\{S x: x \in X_{1}\right\} \subseteq \bigcap\left\{S x: x \in X_{0}\right\} \subseteq K$. This shows that $\cap\left\{S x \cap \overline{T^{\prime}\left(X_{2}\right)} \cap K: x \in N\right\} \neq \emptyset$. Since for each $x \in X, S x$ is compactly closed in $Y$ and $\overline{T^{\prime}\left(X_{2}\right)}$ is compact, it follows that $\left\{S x \cap \overline{T^{\prime}\left(X_{2}\right)} \cap K: x \in X\right\}$ is a family of closed sets with the finite intersection property in the compact set $\overline{T^{\prime}\left(X_{2}\right)} \cap K$. Therefore $\cap\left\{S x \cap \overline{T^{\prime}\left(X_{2}\right)} \cap K: x \in X\right\} \neq \emptyset$. Since $\overline{T^{\prime}\left(X_{2}\right)} \subseteq T\left(X_{2}\right) \subseteq T(X)$, it follows that $K \cap \overline{T(X)} \cap \cap\{S x: x \in X\} \neq \emptyset$.

The following theorem generalises a fixed point theorem of Tarafdar [15].

THEOREM 5. Let $X$ be a convex space, $Y$ a Hausdorff topological space, $T \in$ $K K M(X, Y), F: Y \rightarrow 2^{X}$ be set-valued maps satisfying

(5.1) for each compact set $C$ of $X, \overline{T(C)}$ is compact;

(5.2) for each $y \in T(X), F y$ is a nonempty convex subset of $X$;

(5.3) for each $x \in X, F^{-}(x)$ contains a compactly open subset $O_{x}$ of $Y$;

(5.4) $\bigcup_{x \in X} O_{x}=Y$; and

(5.5) there is a nonempty subset $X_{0} \subset X$ such that $X_{0}$ is contained in a compact convex subset $X_{1}$ of $X$ and the set $M=\bigcap_{x \in X_{0}} O_{x}^{c}$ is compact ( $M$ may be empty) and $O_{x}^{c}$ denotes the complement of $O_{x}$ in $Y$.

Then there exist $\bar{x} \in X$, and $\bar{y} \in T(\bar{x})$ such that $\bar{x} \in F(\bar{y})$.

Proof: For each $x \in X$, we let $S x=O_{x}^{c}$, then $S: X \rightarrow 2^{Y}$ and for each $x \in X$. $S x$ is compactly closed in $Y$. There are two cases:

CASE (1) $M=\emptyset$. In this case, if we take $X=X_{0}$ in Theorem 1, we have a finite subset $A=\left\{x_{1}, x_{2}, \ldots, x_{n}\right\}$ of $X_{0}$ such that $T(\operatorname{Co} A) \nsubseteq \bigcup_{i=1}^{n} S x_{i}$. This means that there exist $x_{0}=$ $\sum_{i=1}^{n} \lambda_{i} x_{i}, \lambda_{i} \geqslant 0, i=1,2, \ldots, n, \sum_{i=1}^{n} \lambda_{i}=1$ and $y_{0} \in T x_{0}$ such that $y_{0} \notin \bigcup_{i=1}^{n} S x_{i}=\bigcup_{i=1}^{n} O_{x_{i}}^{c}$. Thus $y_{0} \in O_{x_{i}} \subseteq F^{-}\left(x_{i}\right)$ for all $i=1,2, \ldots, n$. Hence $x_{i} \in F\left(y_{0}\right)$ for all $=1,2, \ldots, n$. But by (3.1), F $y_{0}$ is convex, so we have $x_{0}=\sum_{i=1}^{n} \lambda_{i} x_{i} \in F y_{0}$ and Theorem 5 is proved for the case $M=\bigcap_{x \in X_{0}} O_{x}^{c}=\emptyset$.

CASE (2) $M \neq \emptyset$. We want to show that there exists a finite subset $A=\left\{x_{1}, x_{2}, \ldots, x_{n}\right\}$ of $X$ such that $T(\mathrm{Co} A) \nsubseteq \bigcup_{i=1}^{n} S x_{i}$. Suppose that for each finite subset $B=\left\{u_{1}, u_{2}, \ldots, u_{m}\right\}$ of $X, T($ Co $B) \subseteq \bigcup_{i=1}^{m} S u_{i}$. Then it follows from Corollary 4 that $\overline{T(X)} \cap \cap\{S x: x \in X\} \neq$ 
$\emptyset$. Hence $\bigcap_{x \in X} O_{x}^{c}=\bigcap_{x \in X} S x \neq \emptyset$, therefore $\bigcup_{x \in X} O_{x} \neq Y$, which contradicts to the assumption (5.4) of this theorem. This shows that there exists a finite subset $A=\left\{x_{1}, x_{2}, \ldots, x_{n}\right\}$ of $X$ such that $T(\operatorname{Co} A) \notin \bigcup_{i=1}^{n} S x_{i}$. As in case (1), there exist $x_{0}=\sum_{i=1}^{n} \lambda_{i} x_{i}, \lambda_{1} \geqslant 0$, $i=1,2, \ldots, n, \sum_{i=1}^{n} \lambda_{i}=1$ and $y_{0} \in T x_{0}$ such that $y_{0} \notin \bigcup_{i=1}^{n} S x_{i}$. From this relation, we get that $x_{0} \in F y_{0}$ and $y_{0} \in T x_{0}$.

Theorem 5 also gives a sufficient conditions for the existence of fixed points for the composition of two set-valued maps.

COROLlary 5. Under the assumption of Theorem 5, there exists $x_{0} \in X$ such that $x_{0} \in F T x_{0}$.

Proof: It follows from Theorem 5, that there exist $x_{0} \in X, y_{0} \in T x_{0}$ such that $x_{0} \in F y_{0}$. Hence $x_{0} \in F T x_{0}$.

COROLlary 6. Let $X$ be a nonempty compact convex subset of a topological vector space, $T \in K K M(X, X)$ and $F: X \rightarrow 2^{X}$ be set-valued maps satisfying

(C6.1) for each $y \in X, F^{-}(y)$ contains a relatively open subset $O_{y}$ of $X\left(O_{y}\right.$ could be empty);

(C6.2) for each $x \in X, F x$ is a nonempty subset of $X$; and

(C6.3) $\bigcup_{y \in X} O_{y}=X$.

Then there exists point $x_{0} \in X, y_{0} \in T x_{0}$ such that $x_{0} \in F y_{0}$.

Proof: Since $X$ is compact and $\bigcup_{y \in X} O_{y}=X$, it follows that condition (5.5) holds automatically and Corollary 6 follows immediately from Theorem 5 .

COROLLARY $7 \cdot[15]$ Let $X$ be a nonempty compact convex subset of a topological vector space. Let $F: X \rightarrow 2^{X}$ be set-valued maps such that

(C7.1) for each $x \in X, F x$ is a nonempty convex subset of $X$;

(C7.2) for each $y \in X, F^{-}(y)$ contains a relatively open subset $O_{y}$ of $X\left(O_{y}\right.$ may be empty for some $y$ );

(C7.3) $\bigcup_{y \in X} O_{x}=X$; and

(C7.4) there exists a nonempty subset $X_{0} \subset X$ such that $X_{0}$ is contained in a compact convex subset $X_{1}$ of $X$ and $M=\bigcap_{x \in X_{0}} O_{x}^{c}$ is compact ( $M$ may be empty).

Then there exists a point $x_{0} \in X$ such that $x_{0} \in F x_{0}$.

Proof: If we define $T: X \rightarrow 2^{X}$ by $T x=\{x\}$ and take $X=Y$ in Theorem 5, we prove Corollary 7. 
Corollary 8. [2] Let $X$ be a nonempty compact convex subset of a topological vector space. Let $F: X \rightarrow 2^{X}$ be set-valued maps such that

(C8.1) for each $y \in X, F^{-}(y)$ is open; and

(C8.2) for each $x \in X, F x$ is a nonempty convex subset of $X$.

Then there is $x_{0} \in X$ such that $x_{0} \in F x_{0}$.

Proof: Since for each $x \in X, F x$ is a nonempty subset of $X$, there exists $y \in X$ such that $y \in F x$. Hence $x \in F^{-} y$. This shows that $X=\bigcup_{y \in X} F^{-} y$. If we define $T: X \rightarrow 2^{Y}$ by $T x=\{x\}$ for $x \in X$, then all the conditions of Corollary 7 are satisfied and Corollary 8 follows immediately from Corollary 7 .

Remark 3. Corollary 4 can be proved by using Theorem 5 . Suppose that all the conditions of Corollary 4 are satisfied; we want to show that $\overline{T(X)} \cap \cap\{S x: x \in X\} \neq \emptyset$. Suppose on the contrary that $\overline{T(X)} \cap \cap\{S x: x \in X\}=\emptyset$. We define $H: \overline{T(X)} \rightarrow 2^{X}$ by $H y=\{x \in X: y \notin S x\}$. For each $x \in X$, we let $S^{c} x=Y \backslash S x$ and $O_{x}=S^{c} x$. Clearly for each $y \in \overline{T(X)}, y \in \bigcup_{x \in X} S^{c} x$, hence $y \notin S x_{0}$ for some $x_{0} \in X$ and $H(y)$ is a nonempty subset of $X$. For each $x \in X, H^{-}(x)=\{y \in \overline{T(X)}: y \notin S x\}=S^{c} x \cap \overline{T(X)}=O_{x} \cap \overline{T(X)}$ is compactly open in $\overline{T(X)}$. Now we denote $\hat{O}_{x}=O_{x} \cap \overline{T(X)}$. Let $F: \overline{T(X)} \rightarrow 2^{X}$ be defined by $F y=\mathrm{Co}[H y]$ for each $y \in \overline{T(X)}$. Then for each $y \in \overline{T(X)}, F y$ is a nonempty convex subset of $X$ and for each $x \in X, F^{-}(x) \supseteq H^{-}(x)=\hat{O}_{x}$. Since $\overline{T(X)} \cap \cap\{S x: x \in X\}=\emptyset$, it follows that $\overline{T(X)} \subset \bigcup_{x \in X} S^{c} x$ and $\overline{T(X)}=\bigcup_{x \in X}\left[S^{c} x \cap\right.$ $\overline{T(X)}]=\bigcup_{x \in X}\left[O_{x} \cap \overline{T(X)}\right]=\bigcup_{x \in X} \widehat{O}_{x}$. We denote by $\widehat{O}_{x}^{c}$ the complement of $\widehat{O}_{x}$ in $\overline{T(X)}$. By (C4.2) and (C4.4), $\bigcap_{x \in X_{0}} \widehat{O}_{x}^{c}=\bigcap_{x \in X_{0}}\left[\overline{T(X)} \backslash \widehat{O}_{x}\right]=\overline{T(X)} \cap \bigcap_{x \in X_{0}} O_{x}^{c}=\overline{T(X)} \cap \bigcap_{x \in X_{0}} S x$ is compact in $\overline{T(X)}$. Then it follows from Theorem 5 that there exists $\bar{x} \in X, \bar{y} \in T(\bar{x})$ such that $\bar{x} \in F \bar{y}=\mathrm{Co}[H \bar{y}]$. This implies there exists $A=\left\{x_{1}, x_{2}, \ldots, x_{n}\right\} \subseteq H(\bar{y}), \lambda_{i} \geqslant 0$, $i=1,2 \ldots, n, \sum_{i=1}^{n} \lambda_{i}=1$ such that $\bar{x}=\sum_{i=1}^{n} \lambda_{i} x_{i}$. Since $x_{i} \in H(\bar{y})$ for all $i=1,2, \ldots, n$, it follows that $\bar{y} \notin S x_{i}$ for all $i=1,2, \ldots, n$. Therefore $T(\operatorname{Co} A) \notin \bigcup_{i=1}^{n} S x_{i}$. This contradicts the assumption (C4.3) of Corollary 4. Hence $\overline{T(X)} \cap \cap\{S x: x \in X\} \neq \emptyset$ and Corollary 4 is proved.

THEOREM 6. Let $X$ be a convex space, $Y$ a Hausdorff topological space, $T \in$ $U_{c}^{\kappa}(X, Y), F: Y \rightarrow 2^{X}$ be set-valued maps satisfying

(6.1) for each $y \in T(X), F y$ is a nonempty convex subset of $X$;

(6.2) for each $x \in X, F^{-}(x)$ contains an compactly open subset $O_{x}$ of $Y$;

(6.3) $\bigcup_{y \in X} O_{x}=Y$; and

(6.4) there exists a nonempty subset $X_{0} \subseteq X$ such that $X_{0}$ is contained in a compact convex subset $X_{1}$ of $X$ and the set $M=\bigcap_{x \in X_{0}} O_{x}^{c}$ is compact ( $M$ may be empty) and $O_{x}^{c}$ denotes the complement of $O_{x}$ in $Y$. 
Then there exist $\bar{x} \in X$ and $\bar{y} \in T \bar{x}$ such that $\bar{x} \in F \bar{y}$.

Proof: For each $x \in X$, we let $S x=O_{x}^{c}$. Then $S: X \rightarrow 2^{Y}$ and for each $x \in X$. $S x$ is compactly closed in $Y$. There are two cases.

CASE (1) $M=\emptyset$. In this case, we use Corollary 1 and follow the same argument as in Theorem 5 .

CASE (2) $M \neq \emptyset$. In this case, we use Theorem 4 and follow the same argument as in Theorem 5 .

REMARK 4. In Theorem 5, we assume that $T \in K K M(X, Y)$ and $\overline{T(C)}$ is compact for each compact set $C$ of $X$, but in Theorem 6, we assume only that $T \in U_{c}^{\kappa}(X, Y)$.

THEOREM 7. Let $(X, D ; \Gamma)$ be a $G$-convex space, $Y$ a Hausdorff space, and $T$ : $X \rightarrow 2^{Y}$ be compact and closed and $G: D \rightarrow 2^{Y}$. Suppose that

(7.1) for each $x \in D, G x$ is compactly closed;

(7.2) for any $N \in\langle D\rangle, T\left(\Gamma_{N}\right) \subseteq G(N)$; and

(7.3) there exist a nonempty compact subset $K$ of $Y$ and for each $N \in\langle D\rangle$, a compact, $G$-convex subset $L_{N}$ of $X$ containing $N$ such that $T\left(L_{N}\right) \cap \cap\{G x$ : $\left.x \in L_{N} \cap D\right\} \subset K$, and $T \in G-K K M\left(L_{N}, Y\right)$.

Then $\overline{T(X)} \cap K \cap \cap\{G x: x \in D\} \neq \emptyset$.

Proof: Suppose that $\overline{T(X)} \cap K \cap \cap\{G x: x \in D\}=\emptyset$. Let $S x=Y \backslash G x$, then $\overline{T(X)} \cap K \subset S(D)$. Since $\overline{T(X)} \cap K$ is compact and for each $x \in D, S x$ is compactly open, it follows that there exists $N \in\langle D\rangle$ such that $\overline{T(X)} \cap K \subseteq S(N)$. By (7.3), there exists a compact $G$-convex subset $L_{N}$ of $X$ containing $N$ such that $T\left(L_{N}\right) \backslash K \subseteq S\left(L_{N} \cap D\right)$. Hence $T\left(L_{N}\right) \subseteq S\left(L_{N} \cap D\right)$. Since $T$ is compact and closed, it follows from Lemma 2 that $T$ is upper semicontinuous We want to show that for each $x \in X, T x$ is compact. Let $y \in \overline{T(x)}$, then there exists a net $\left\{y_{\alpha}\right\}$ in $T x$ such that $y_{\alpha} \rightarrow y$. Since $T$ is closed, it follows that $y \in T x$ and $T x$ is closed. By assumption $T$ is compact, hence $\overline{T(X)}$ is a compact set. But $T x \subseteq \overline{T(X)}$ and $T x$ is closed for each $x \in X$. This shows that $T x$ is compact for each $x \in X$. Since $T$ is upper semicontinuous with compact values and $L_{N}$ is compact, it follows from Lemma 3 that $T\left(L_{N}\right)$ is compact. Therefore $\overline{T\left(L_{N}\right)}=T\left(L_{N}\right) \subseteq S\left(L_{N} \cap D\right)$. Thus $\overline{T\left(L_{N}\right)} \cap \cap\left\{G x: x \in L_{N} \cap D\right\}=\emptyset$. It follows from Theorem 1 with $\left(\left.T\right|_{L_{N}},\left.G\right|_{L_{N} \cap D}, L_{N}, L_{N} \cap D\right)$ replacing $(T, G, X, D)$, that there exists $M \in\left\langle L_{N} \cap D\right\rangle \subseteq\langle D\rangle$ such that $T\left(\Gamma_{M}\right) \nsubseteq G(M)$. This contradicts (7.2). Therefore $\overline{T(X)} \cap K \cap \cap\{G: x \in D\} \neq \emptyset$.

Corollary 9. [12] Let $(X, D ; \Gamma)$ be a G-convex space, $Y$ a Hausdorff space, and $T \in U_{c}^{\kappa}(X, Y)$. Let $G: D \rightarrow 2^{Y}$ be a map such that

(C9.1) for each $x \in D, G x$ is compactly closed in $Y$;

(C9.2) for any $N \in\langle D\rangle, T\left(\Gamma_{N}\right) \subseteq G(N)$; and 
(C9.3) there exist a nonempty compact subset $K$ of $Y$ and for each $N \in\langle D\rangle$, a compact $G$-convex subset $L_{N}$ of $X$ containing $N$ such that $T\left(L_{N}\right) \cap \cap\{G x$ : $\left.x \in L_{N} \cap D\right\} \subset K$.

Then $\overline{T(X)} \cap K \cap \cap\{G x: x \in D\} \neq \emptyset$.

Proof: Since $T \in U_{c}^{\kappa}(X, Y) \subset G-K K M(X, Y)$, it follows from Lemma 6 that $\left.T\right|_{L_{N}} \in G-K K M\left(L_{N}, Y\right)$ and the conclusion of Corollary 9 follows from Theorem 7 .

\section{Generalised G-KKM theorems}

As a consequence of the generalised $G-K K M$ theorem, we prove a generalisation of the Ky Fan matching theorem.

THEOREM 8. Let $(X, D ; \Gamma)$ be a $G$-convex space, $Y$ a Hausdorff space, $S: D \rightarrow$ $2^{Y}$ and $T \in G-K K M(X, Y)$ be compact. Suppose that

(8.1) for each $x \in D, S x$ is compactly open in $Y$; and

(8.2) $\overline{T(X)} \subset S(D)$.

Then there exists $M \in\langle D\rangle$ such that $T\left(\Gamma_{M}\right) \cap \cap\{S x: x \in M\} \neq \emptyset$.

Proof: Suppose that the conclusion of Theorem 8 is false. Then for any $N \in\langle D\rangle$, $T\left(\Gamma_{N}\right) \cap \cap\{S x: x \in N\}=\emptyset$. Therefore $T\left(\Gamma_{N}\right) \subseteq \cup\{G s: s \in N\}=G(N)$, where $G x=$ $Y \backslash S x$. By (8.1), for each $x \in D, G x$ is compactly closed in $Y$. Then all the conditions of Theorem 1 are satisfied. It follows from Theorem 1 that $\overline{T(X)} \cap \cap\{G x: x \in D\} \neq \emptyset$. Hence $\overline{T(X)} \nsubseteq S(D)$, but this contradicts (8.2). Thus there exists $M \in\langle D\rangle$ such that $T\left(\Gamma_{M}\right) \cap \cap\{S x: x \in M\} \neq \emptyset$.

COROLlary 10. [8] Let $D$ be a nonempty subset in a compact convex space $X, Y$ a topological space, and $A: D \rightarrow 2^{Y}$ a set-valued map satisfying

(C10.1) for each $x \in D, A x$ is compactly open in $Y$; and

(C10.2) $\quad A(D)=Y$.

Then for any $x \in C(X, Y)$, there exist a finite subset $\left\{x_{1}, x_{2}, \ldots, x_{n}\right\}$ of $X$ and $x_{0} \in$ Co $\left\{x_{1}, \ldots, x_{n}\right\}$ such that $s x_{0} \in \bigcap_{i=1}^{n} A x_{i}$.

Proof: Since $X$ is compact and $s \in C(X, Y)$, it follows that $s(X)$ is compact. Hence $s \in C(X, Y) \subseteq K K M(X, Y)$ is compact. By $(\mathrm{C} 10.2), \overline{s(X)}=s(X) \subseteq Y \subseteq A(D)$. It follows from Theorem 8 , that there exist a finite subset $\left\{x_{1}, x_{2}, \ldots, x_{n}\right\}$ of $X$ and $x_{0} \in \mathrm{Co}\left\{x_{1}, \ldots, x_{n}\right\}$ such that $s x_{0} \in \bigcap_{i=1}^{n} A x_{i}$.

COROLLARY 11. [5] In a topological vector space, let $Y$ be a convex set and let $X$ be a nonempty subset of $Y$. For each $x \in X$, let $A x$ be relative open in $Y$ such that $\bigcup_{x \in X} A x=Y$. If $X$ is contained in a compact convex subset $C$ of $Y$, then there exist a nonempty, finite subset $\left\{x_{1}, x_{2}, \ldots, x_{n}\right\}$ of $X$ and $x_{0} \in\left\{x_{1}, \ldots, x_{n}\right\}$ such that $x_{0} \in \bigcap_{i=1}^{n} A x_{i}$. 
PRoOF: Let $T x=\{x\}$, then $T(C)=C$ is compact, and $T$ is compact. $\overline{T(C)}=$ $C \subseteq Y \subseteq A(X)$. Then it follows from Theorem 8 that there exist a finite subset $\left\{x_{1}, x_{2}, \ldots, x_{n}\right\}$ of $X$ and $x_{0} \in\left\{x_{1}, \ldots, x_{n}\right\}$ such that $x_{0} \in \bigcap_{i=1}^{n} A x_{i}$.

REMARK 5. Theorems 1 and 8 are equivalent.

We saw that Theorem 8 can be proved by using Theorem 1 . Now we prove Theorem 1 from Theorem 8. Suppose that $\overline{T(X)} \cap \cap\{G x: x \in D\}=\emptyset$. Let $S x=Y \backslash G x$. Then $S x$ is compactly open and $\overline{T(X)} \subset S(D)$. It follows from Theorem 8 , that there exists $M \in\langle D\rangle$ such that $T\left(\Gamma_{M}\right) \cap \cap\{S x: x \in M\} \neq \emptyset$. Hence $T\left(\Gamma_{M}\right) \nsubseteq G(M)$. This contradicts (1.2). Thus the conclusion of Theorem 1 holds.

Theorem 9. Let $(X, D ; \Gamma)$ be a $G$-convex space, $Y$ a Hausdorff space and $T$ : $X \rightarrow 2^{Y}$ be compact and closed. Suppose that

(9.1) for each $x \in D, S x$ is compactly open;

(9.2) there exists a nonempty compact subset $K$ of $Y$ such that $\overline{T(X)} \subset S(D)$; and

(9.3) for each $N \in\langle D\rangle$, there exists a compact $G$-convex subset $L_{N}$ of $X$ containing $N$ such that $T\left(L_{N}\right) \backslash K \subseteq S\left(L_{N} \cap D\right)$, and $T \in G-K K M\left(L_{N}, Y\right)$.

Then there exists $M \in\langle D\rangle$ such that $T\left(\Gamma_{M}\right) \cap \cap\{S x: x \in M\} \neq \emptyset$.

Proof: Suppose that for any $N \in\langle D\rangle . T\left(\Gamma_{N}\right) \cap \cap\{S x: x \in N\}=\emptyset$. Let $G x=Y \backslash S x$. Then by applying Corollary 9 and following an argument as in Theorem 8, we prove Theorem 9 .

\section{REFERENCES}

[1] J.P. Aubin, A. Cellina, Differential inclusions (Springer-Verlag, Berlin, Heidlberg, New York, 1984).

[2] F.E. Browder, 'On nonlinear monotone operators and convex set in Banach space', Bull. Amer. Math. Soc. 71 (1965), 780-785.

[3] T.H. Chang and C.L. Yen, 'KKM property and fixed point theorems', J. Math. Anal. Appl. 203 (1996), 224-235.

[4] K. Fan, 'A generalization of Tychonoff's fixed point theorem', Math. Ann. 142 (1961), 306-310.

[5] K. Fan, 'Some properties of convex sets related to fixed point theorem', Math. Ann. 266 (1985), 519-537.

[6] B. Knaster, K. Kuratowski and S. Mazurkiewicz, 'Ein Beweis Fixpunktsatzes fur n-dimensionale Simplexe', Fund. Math. 1 (1929), 132-137.

[7] M. Lassonde, 'On the use of KKM multifunctions in fixed point theory and related topics', J. Math. Anal. Appl. 97 (1983), 151-201.

[8] S. Park, 'Generalizations of Ky Fan's matching theorems and their applications', $J$. Math. Anal. Appl. 141 (1989), 164-176. 
[9] S. Park, 'A unfied approach to generalizations of the KKM-type theorems related to acyclic maps', Numer. Funct. Anal. Optim. 15 (1994), 105-119.

[10] S. Park, 'Foundations of the KKM theory via coincidence of composites of upper semicontinuous maps', J. Korean Math. Soc. 31 (1994), 493-519.

[11] S. Park, 'On minimax inequalities on spaces having certain contractible subsets', Bull. Austral. Math. Soc. 47 (1993), 25-40.

[12] S. Park and H. Kim, 'Coincidence theorems for admissible multifunctions on generalized convex spaces', J. Math. Anal. Appl. 197 (1996), 173-187.

[13] S. Park and H. Kim, 'Fundations of the KKM theory on generalized convex spaces', $J$. Math. Anal. Appl. 209 (1997), 551-571.

[14] N. Shioji, 'A further generalization of the Knaster-Kuratowski-Mazurkiewicz theorem', Proc. Amer. Math. Soc. 111 (1991), 187-195.

[15] E. Tarafdar, 'A fixed point theorem equivalent to the Fan-Knaster-KuratwskiMazurkiewicz theorem', J. Math. Anal. Appl. 128 (1987), 475-479.

[16] E. Tarafdar, 'On nonlinear variational inequalities', Proc. Amer. Math. Soc. 67 (1977), 95-98.

Department of Mathematics

National Changhua University of Education

Changhua

Taiwan

Republic of China 